the degradation of D-leucine more than of L-leucine; and what is most striking, right-handed electrons decomposed Lleucine more than the D-isomer. Thus, the "natural" electrons produced an asymmetric degradation favouring the "natural" amino acid, and vice versa.

From the results reported in the last few months it seems reasonable to conclude that $\beta$ rays can indeed induce optical activity. What remains totally obscure is the mechanism; for example, in the experiments by Bonner et al., was the effect a direct one of the polarised electrons themselves, or was it due to bremsstrahlung?

\section{Transposable resistance genes}

\section{from J. R. Saunders}

It is increasingly evident that certain bacterial resistance genes are capable of migration between DNA molecules. This ability makes it easier to understand the rapid evolution of antibioticresistance (R) plasmids possessing varied combinations of linked resistance genes and may indicate a more general evolutionary process in bacteria and other organisms.

The curious ability of a gene specifying ampicillin-resistance to be translocated to different replicons in bacteria has been known for some time (see, for example, Anderson, CIBA Symposium on Bacterial Episomes and Plasmids, 102, 1969; Richmond and Sykes, Genet. Res., 20, 231; 1972). Hedges and Jacob (Molec. gen. Genet., 132, 31; 1974) demonstrated that the ampicillin-resistance gene present on the plasmid RP4 can be acquired by a variety of other plasmids co-existing in the same strain of Escherichia coli. plasmids acquiring this gene showed concomitant increases in molecular weight, and were in turn able to translocate the gene, which they termed Transposon A ( $\operatorname{TnA}$ ), to other plasmids. Recently a number of other resistance genes capable of translocation between chromosomal, phage and plasmid DNA have been identified. These include, for example, kanamycin-resistance (Berg et al., Proc. natn. Acad. Sci. U.S.A., 72, 3628; 1975), tetracycline-resistance (Kleckner et al., J. molec. Biol., 97, 561; 1975) and linked resistance to streptomycin and trimethoprim (Hedges et al., $\mathrm{Mol}$. gen. Genet., 140, 289; 1975). This suggests that discrete translocatable elements (transposons) may constitute relatively common genetic units in bacteria.

Transposons are apparently able to insert into and excise from DNA molecules in the absence of a functional bacterial recombination $(\operatorname{rec} A)$ system. This suggests that such elements use alternative, possibly selfspecified enzymes for recombination. Despite this partial independence, transposons have not been shown to exist autonomously and need to reside in a functional replicon.

A clue to the mechanism of insertion and excision comes from electron microscope studies of DNA heteroduplexes formed between phage or plasmid DNA with and without inserted transposons. These indicate that most transposons are composed of a resistance gene (or genes) flanked on either side by inverted complementary DNA sequences (inverted repetitions) of between 100 and 1,500 nucleotide pairs. Some, but not all such repetitions are homologous with known insertion sequences (IS). For example, the inverted repetitious sequences bounding the transposable tetracycline-resistance gene are homologous with IS3. (Ptashne and Cohen, J. Bact., 122, 776; 1975). Insertion sequences are present at various sites in both plasmids and the chromosome of $E$. coli ( $\mathrm{Hu}$ et al., J. Bact., 122, $764 ; 1975)$ and have been implicated in a number of rec $A$-independent and illegitimate recombination events.

Insertions of transposons are mutagenic when occurring in structural genes and polar when occurring within operons. This is also a general property of insertion sequences (Starlinger and Saedler, Biochimie, 54, 177; 1972). As shown by Klechner et al. insertion itself is a relatively precise process which does not damage the informational capacity of host DNA. On the other hand, excision often results in permanent loss of host genetic information. Heffron and colleagues (Proc. natn. Acad. Sci. U.S.A., 72, 3623 ; 1975) show that $\operatorname{TnA}$ is able to insert at a minimum of twelve distinct sites on the genome of the plasmid RSF1010, suggesting recognition by the transposon of a specific but fairly common nucleotide sequence at or near to the point of integration. Insertion/excision must be by a mechanism which conserves the inverted repetitions since transposons can be repeatedly translocated whilst retaining such sequences. This could occur if excision involves recombination between the two flanking inverted repetitions such that one of the 'arms' of the repeat is retained by the genome and the other by the looped-out resistance gene. The latter could then circularise and migrate either to another DNA molecule or to a different site on the same molecule. Provided that a homologous repeat sequence is present on the recipient molecule insertion is then possible by recombination between the two repeats. It should be pointed out however, that circularised transposons have not yet been detected in the process of migration, which suggests that such a stage, if present, is short-lived.

The significance of transposition is that it allows rearrangement and recombination of certain genes from heterologous genetic backgrounds. The phenomenon also provides a neat mechanism for the accretion of resistance genes by sex factors to form infectious multiple resistance plasmids. It is known that relatively few types of resistance gene are common to a wide variety of $R$ plasmids, found in different bacterial species, and believed on both genetic and physical grounds to be of disparate evolutionary origin. For example, the $\beta$-lactamase (penicillinase) gene of $\operatorname{Tn} A$, and base sequences homologous with it, are widely distributed on an assortment of plasmids (Heffron et al., J. Bact., 122, $250 ; 1975)$. This suggests that all have received the resistance gene from a common source. Transposons presumably evolved initially in their ancestral host by association of resistance genes with inverted repetitious sequences. Such elements could then be attached to a variety of replicons, including genetic vectors and hence find their way to other bacterial strains where they could be retransposed to resident DNA molecules. This process is of enormous evolutionary advantage because it obviates the need for the gross genetic homology normally required for recombination and allows reassortment of genes on different replicons. Therefore tandem insertion of numbers of resistance genes into a plasmid of unrelated nucleotide sequence would be possible, provided that the relatively short relevant inverted repetitions were present.

Transposons may prove powerful tools for genetic research since attachment of a resistance gene to a DNA molecule provides it with a readily discernible genetic and physical tag. It will be of great interest to discover the precise mechanism of their insertion and excision. Ways of doing this would include sequencing of the inverted repeats, particularly at their functions with non-repetitious DNA, and searching for a specific enzymatic machinary for mediating insertion and excision. It seems likely that the latter will involve enzymes with the specificity of restriction nucleases for recognising insertion sites. In view of the frequency of inverted repetitious DNA in both prokaryotes and eukaryotes, transposons may represent a more general class of genetic elements contributing greatly to evolutionary flexibility. 